\title{
Occurrence and transport of 17 perfluoroalkyl acids in 12 coastal rivers in south Bohai coastal region of China with concentrated fluoropolymer facilities
}

\author{
Pei Wang ${ }^{\mathrm{a}, \mathrm{b}}$, Yonglong Lu ${ }^{\mathrm{a}, *}$, Tieyu Wang ${ }^{\mathrm{a}}$, Yaning Fu ${ }^{\mathrm{a}, \mathrm{b}}$, Zhaoyun Zhu ${ }^{\mathrm{a}, \mathrm{b}}$, Shijie Liu ${ }^{\mathrm{a}, \mathrm{b}}$, \\ Shuangwei Xie ${ }^{\mathrm{a}, \mathrm{b}}$, Yang Xiao ${ }^{\mathrm{a}, \mathrm{b}}$, John P. Giesy ${ }^{\mathrm{c}}$ \\ ${ }^{a}$ State Key Lab of Urban and Regional Ecology, Research Center for Eco-Environmental Sciences, Chinese Academy of Sciences, Beijing 100085, China \\ ${ }^{\mathrm{b}}$ Graduate University of Chinese Academy of Sciences, Beijing 100049, China \\ ${ }^{\mathrm{c}}$ Department of Veterinary Biomedical Sciences and Toxicology Centre, University of Saskatchewan, Saskatoon, Saskatchewan, Canada
}

\section{A R T I C L E I N F O}

\section{Article history:}

Received 4 January 2014

Received in revised form 19 March 2014

Accepted 21 March 2014

Available online 17 April 2014

\section{Keywords:}

Perfluoroalkyl acids

PFOA

Spatial analysis

Rapid urbanization

Fluoropolymer facility

\begin{abstract}
A B S T R A C T
Perfluoroalkyl acids (PFAAs) are emerging contaminants that have raised great concern in recent years. While PFAAs manufacturing becomes regulated in developed countries, production has been partly shifted to China. Eight fluoropolymer manufacturing facilities located in the South Bohai coastal region, one of the most populated areas of China, have been used to manufacture PFAA-related substances since 2001. The environmental consequence of the intensive production of PFAAs in this region remains largely unknown. We analyzed 17 PFAAs in twelve coastal rivers of this region, and found staggeringly high concentrations of perfluorooctanoic acid (PFOA) ranging from 0.96 to $4534.41 \mathrm{ng} / \mathrm{L}$. The highest concentration was observed in the Xiaoqing River which received effluents from certain fluoropolymer facilities. Principal component analysis indicated similar sources of several perfluoroalkyl carboxylic acids (PFCAs) in all rivers, which indicated that atmospheric transport, wastewater treatment and surface runoff also acted as important supplements to direct discharge to surface water.
\end{abstract}

(C) 2014 Elsevier Ltd. All rights reserved.

\section{Introduction}

Perfluoroalkyl acids (PFAAs) have unique properties including surface activity, repellency of water and oil, and resistance of acid and heat (Giesy and Kannan, 2002). In industry, they are widely used in manufacturing processes and products (Giesy et al., 2006), but when dispersed into the environment, they can transport over long distances and accumulate to toxic concentrations, due to their persistence (Giesy et al., 2010). Ionic PFAAs, mostly known as perfluoroalkyl carboxylic acids (PFCAs) and perfluoroalkyl sulfonic acids (PFSAs), are relatively soluble compared to many of the organochlorine compounds with similar molecular size such as DDT and Hexachlorocyclohexane. Thus, water is the primary reservoir of PFAAs and the major medium for their transportation (Prevedouros et al., 2005).

Concerns over sources, transport and fate of PFAAs in the aquatic environment have grown rapidly in recent years.

\footnotetext{
* Corresponding author.

E-mail address: yllu@rcees.ac.cn (Y. Lu).
}

Perfluorooctane sulfonic acid (PFOS) is the most frequently detected PFAA that is released from a variety of diffuse sources, and is usually the predominant PFAAs detected in aquatic biota (Giesy et al., 2010). Perfluorooctanoic acid (PFOA) is discharged primarily from point-sources in industrial regions, especially from manufacturing facilities (Pistocchi and Loos, 2009). Wastewater treatment plants (WWTPs) are also important point sources of releasing PFAAs, along with more diffuse inputs including rain, dry deposition and release during the use of products (Muller et al., 2011). The majority of PFAAs reach the coastal marine environment dissolved in water, while for long chain PFCAs (C12C15), about a half of the load was absorbed to particles during transportation (Zushi et al., 2012). Higher affinity for organic carbon leads to enhanced sorption of longer chain PFCAs to particles and solids in sediment and sludge (Armitage et al., 2009). The transportation of PFAAs in water indicated that rivers would be the main source of PFAAs for coastal water. Due to the restriction on the production and use of C8 PFOS and PFOA, the C4 and $C 6$ chemicals have been developed well to adequately replace most current $\mathrm{C} 8$ and higher homologues. However, this has also led to the emergence of short chain PFAAs in the environment 
(Moller et al., 2010; Oliaei et al., 2013). This suggested the importance of the measurement of PFAAs with different carbon chain lengths with different properties, in order to trace the trend of PFAAs contamination in the environment.

The Bohai-Rim Economic Circle is a highly urbanized and industrialized region in Northern China (Fig. S1). There are more than 40 rivers flowing into the Bohai Sea, a semi-enclosed sea. Based on estimation of mass fluxes of several chemical pollutants (i.e. petroleum hydrocarbons, heavy metals) to the Bohai Sea, the rivers contributed $50 \%-70 \%$ of the total inventory among the five sources: rivers, drains, atmospheric deposition, cultivation and non-point sources (Wang and Li, 2006). The authors have conducted systematic studies on PFAAs in the northern part of the Bohai coastal region since 2008, and found that PFAAs were widely distributed in the environmental matrices with PFOA dominant in the Northern Bohai coastal region (Wang et al., 2011b), PFOS was dominant in the aquatic products in Tianjin (Chen et al., 2011), PFAAs concentrations in surface water were correlated with the level of industrialization in Northern China (Wang et al., 2011a), Fluoropolymer production in the northern Bohai coastal region had posed potential impacts to local soils (Wang et al., 2013b), and the PFOS emissions from industrial and domestic sources in the eastern coastal region of China were identified and estimated (Chen et al., 2009; Xie et al., 2013a, 2013b). Results of the studies conducted by other researchers in the northern part also indicated the presence of great concentrations of PFAAs in river water, sediment, soil, precipitation, organisms and human blood from various sources including fluorine industry parks (Jin et al., 2007; Bao et al., 2009, 2010; Liu et al., 2009; Li et al., 2011; Pan et al., 2011). In recent years, urbanization has also sped up in the southern part of the Bohai coastal region, while information on the concentrations of PFAAs in the rapidly urbanized southern portion of Bohai-Rim is still limited.

This study is an extension of our research on tracing the source and fate of PFAAs from adjacent riverine and estuarine areas of the Bohai Sea. The major aim was to investigate the key species of PFAAs in southern Bohai coastal region, and to identify their potential sources and fate. With less usage and strict control over emissions of PFOS in the world, it is still necessary to measure the whole series of known PFCAs and PFSAs in surface water for understanding the status and trends of their production and presence in the environment. Spatial analysis of integrated geographic information will help to discover potential effluents and understand fate and transport from sources to surface water and from rivers to the sea. The hydrological cycle between land and ocean brings PFAAs from the land to the sea through river flow. Thus, the quantification of PFAAs loading in each river to the southern Bohai Sea will contribute information not only to understanding the behavior of PFAAs, but also to more accurate modeling of trace contaminants in these systems for estimating potential risks.

\section{Materials and methods}

2.1. Collection of water samples

Twelve coastal rivers within latitude $38^{\circ} 22^{\prime}$ north and longitude $120^{\circ} 44^{\prime}$ east, which flow into the Bohai Sea from the south were selected. For each river, at least 2 sites were chosen considering different volumes of discharge at distances of 20$30 \mathrm{~km}$ from the estuary (Fig. S1). In September 2011, 35 samples of surface water were collected using $1 \mathrm{~L}$ polypropylene bottles, stored in icebox during transportation and left in room temperature as all samples were extracted within one week after arrival in the lab, the left was stored at $-20{ }^{\circ} \mathrm{C}$ for long-term storage. Parameters including turbidity, $\mathrm{pH}$, dissolved oxygen, conductance, water temperature, concentrations of chloride ion and fluorine ion, and salinity were measured in situ using a HQd Portable and Benchtop Meter Configurator (HACH Company, USA) (Table S2). Before analysis, all the samples were left to stand for $24 \mathrm{~h}$ to settle nonsuspended substances, and $400 \mathrm{~mL}$ supernatant for each sample was taken for the following analysis.

\subsection{Extraction, identification and quantification of target analytes}

17 PFAAs, including 13 PFCAs with carbon lengths from C4 to C18, and 4 PFSAs were identified and quantified (Table S3). A $400 \mathrm{~mL}$ aliquot of unfiltered water was extracted using OASIS WAX-SPE following published methods (Taniyasu et al., 2005) with some modifications. Briefly, prior to loading samples, the Oasis WAX cartridges ( $6 \mathrm{cc}, 150 \mathrm{mg}, 30 \mu \mathrm{m}$, Waters, Milford, MA) were preconditioned with $4 \mathrm{~mL}$ of $0.1 \% \mathrm{NH}_{4} \mathrm{OH}$ in methanol, $4 \mathrm{~mL}$ of methanol, and $4 \mathrm{~mL}$ of Milli-Q water After loading the samples, cartridges were washed with $4 \mathrm{~mL} 25 \mathrm{mM}$ ammonium acetate ( $\mathrm{pH} 4$ ) and air-dried overnight. Target analytes were then eluted with $4 \mathrm{~mL}$ of methanol and $4 \mathrm{~mL}$ of $0.1 \% \mathrm{NH}_{4} \mathrm{OH}$ in methanol, respectively. The latter fraction was reduced to $0.5 \mathrm{~mL}$ under high purity nitrogen (99.999\%, Haidian District, BJ) and passed through a nylon filter ( $13 \mathrm{~mm}, 0.2 \mu \mathrm{m}$, Chromspec, Ontario, Canada), then transferred into a $1.5 \mathrm{~mL}$ PP snap top brown glass vial with polyethylene (PE) septa. Individual PFAA was separated and quantified via Agilent 1290 Infinity HPLC System coupled to an Agilent 6460 Triple Quadrupole LC/MS System (Agilent Technologies, Palo Alto, CA) that was operated in the negative electrospray ionization (ESI) mode. Conditions under which the instrument was operated were listed in Table S4.

\section{3. $Q A / Q C$}

Field blanks, transport blanks, procedure blanks and solvent blanks were conducted with every sample set. External standard curves of 9-point ranging from $0.01 \mathrm{ng} / \mathrm{mL}$ to $100 \mathrm{ng} / \mathrm{mL}$ were prepared for quantification of individual PFAA with coefficients $\left(r^{2}\right)$ for all target analytes exceeding 0.99 . The limit of detection (LOD) and limit of quantification (LOQ) were defined as the peak of analyte that needed to yield a signal-to-noise $(\mathrm{S} / \mathrm{N})$ ratio of $3: 1$ and $10: 1$, respectively. Matrix spike recoveries ranged from $75 \%$ to $126 \%$, while procedure recoveries ranged from $77 \%$ to $122 \%$. Concentrations of PFAAs were not corrected for recoveries. Detailed QA/QC measurements of PFAAs in water were given in the Supplementary data.

\subsection{Statistical and spatial analysis}

Statistical analysis was performed by use of SPSS Statistics V20.0 (SPSS Inc. Quarry Bay, HK). During the analysis, values of concentrations less than the LOQ were set to one-half of the LOQ, and those less than the LOD were assigned values of LOD $/ \sqrt{2}$ (Bao et al., 2010). Prior to the principal component analysis (PCA), tests of normality were carried out to ensure that data met the assumptions used for further analysis. More details on PCA were given in the Supplementary data. Spatial distributions of PFAAs were analyzed using the Arcmap module in ArcGIS V10.0 software (ESRI, Redland, CA). Layers including Digital Elevation Model (DEM) of land and sea, land use and vegetation were obtained from the National Geomatics Center of China (Haidian District, BJ).

\section{Results and discussion}

\subsection{PFAAs in South Bohai coastal rivers}

Among the 17 PFAAs quantified, the concentrations of perfluorododecanoic acid (PFDoA), perfluorotridecanoic acid (PFTrDA), perfluorotetradecanoic acid (PFTeDA), perfluorohexadecanoic acid (PFHxDA), Perfluorooctadecanoic acid (PFODA) and perfluorodecanesulfonate (PFDS) were less than the LOQ in all samples, therefore they were not discussed further in this or the following sections. Concentrations of the remaining PFAAs were listed in Fig. 1 and Table S5.

PFAAs were detected in all the rivers with concentrations of sum PFAAs ( $\sum$ PFAAs) ranging from 2.21 to $5068.97 \mathrm{ng} / \mathrm{L}$. In the Xiaoqing River, PFOA was the dominant PFAA with a mean concentration of $3112.28 \mathrm{ng} / \mathrm{L}$, which contributed $90.1 \%$ of the $\sum$ PFAAs, and was followed by short chain PFCAs, including PFBA (mean concentration of $49.80 \mathrm{ng} / \mathrm{L}, 1.4 \%$ ), PFPeA (mean concentration of $70.97 \mathrm{ng} / \mathrm{L}$, 2.0\%), PFHxA (mean concentration of $123.34 \mathrm{ng} / \mathrm{L}, 3.5 \%$ ) and PFHpA (mean concentration of $91.72 \mathrm{ng} / \mathrm{L}, 2.6 \%$ ). For concentrations of the other long chain PFCAs and all PFSAs, the total contribution was less than $1 \%$. In the remaining 11 rivers, the mean concentration of $\sum$ PFAAs was $25.78 \mathrm{ng} / \mathrm{L}$ with the average contribution of individual PFAA in decreasing order of percentage: PFOA (38.2\%) > PFBA $(19.5 \%)>\operatorname{PFOS}(14.3 \%)>\operatorname{PFHxA}(6.7 \%)>\operatorname{PFPeA}(6 \%)>\mathrm{PFBS}$ (3.9\%) $>$ PFNA $(4.6 \%)>$ PFHpA $(4.3 \%)>$ PFDA.

(1.1\%) $>$ PFHxS (1\%) > PFUdA (0.2\%) (Fig. 2a). PCA analysis on the 11 PFAAs and 9 water parameters showed that PFBA, PFPeA, PFHxA, and PFOA were associated when the Xiaoqing River was excluded 


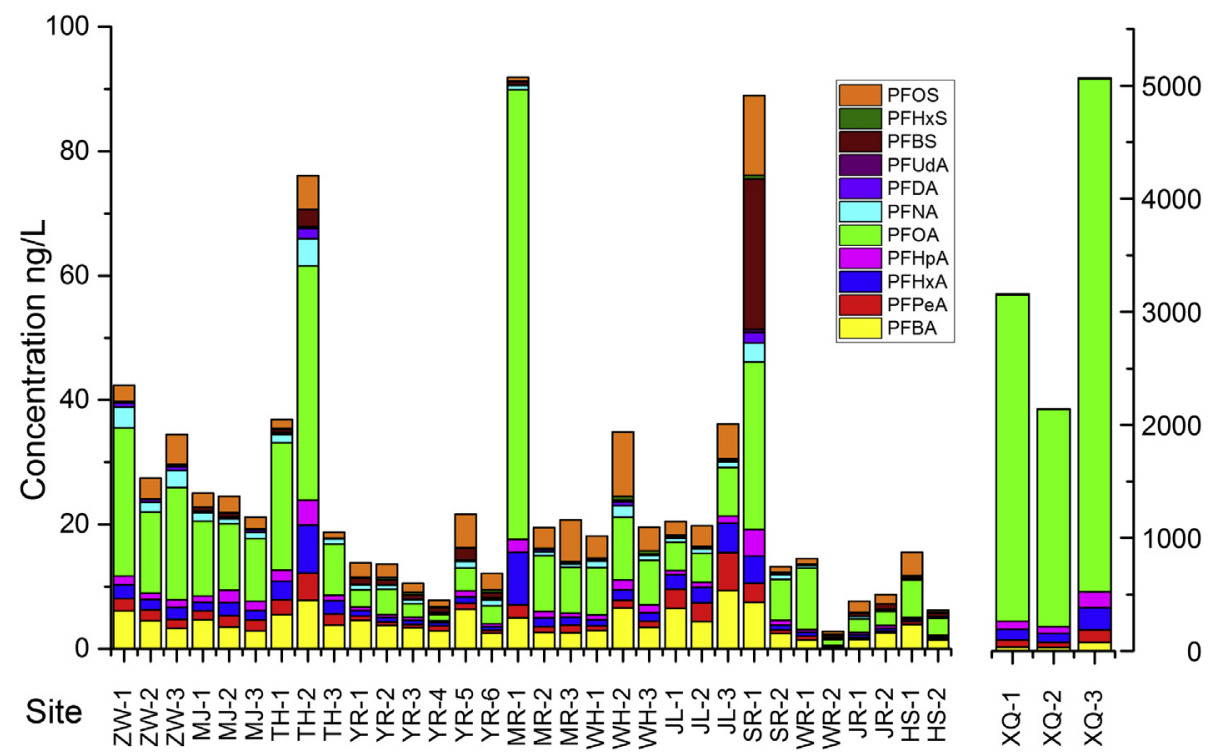

Fig. 1. Concentrations of the 11 PFAAs (ng/L) in South Bohai coastal rivers.

due to the extremely high levels of PFOA (Fig. 2b). When the Xiaoqing River was included, the association among the four PFCAs became much stronger (Fig. 2c). This indicated that these compounds might come from similar sources. However, there were still differences in the two scenarios, which might explained by the different weights of various sources to the rivers. Concentrations of
PFOS ranged from 0.40 to $12.78 \mathrm{ng} / \mathrm{L}$ with a mean concentration of $3.09 \mathrm{ng} / \mathrm{L}$ for these rivers. The highest concentration of PFOS was in SR-1, where concentrations of PFBS (24.19 ng/L) and PFHxS ( $0.59 \mathrm{ng} / \mathrm{L})$ were also highest. SR-1 was located in an estuary where there might be local releases of PFSAs (Table S1). Furthermore, concentrations of PFOS and PFBS were always correlated in the two (a)

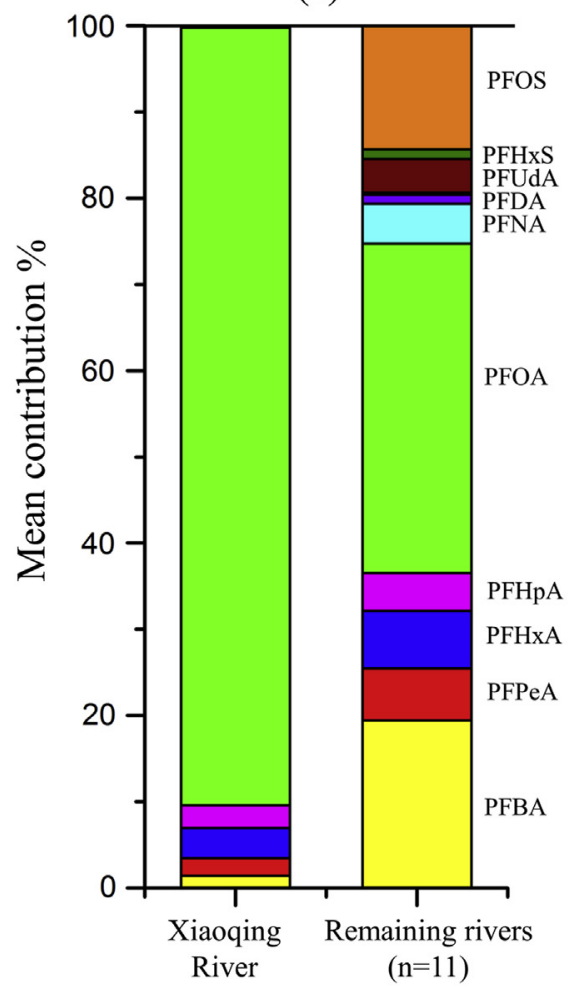

(b)

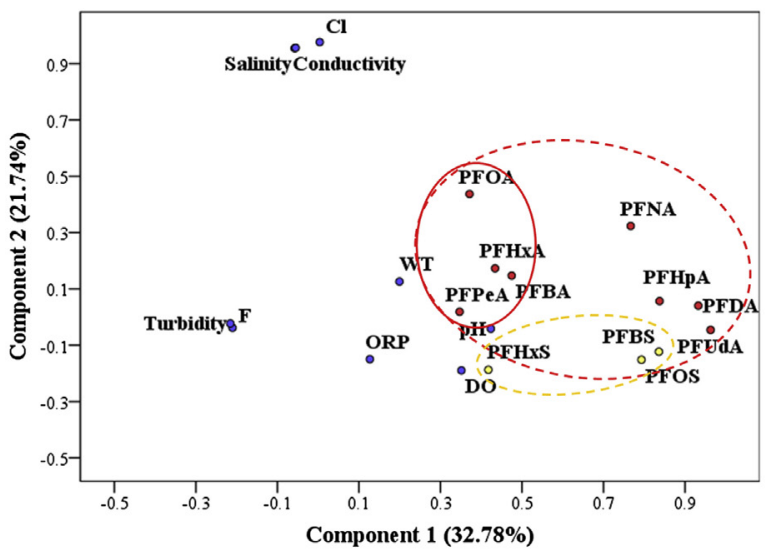

(c)

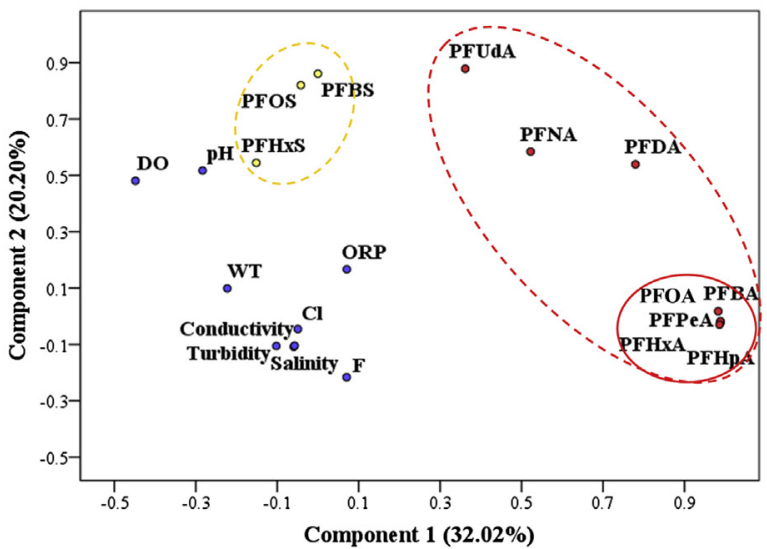

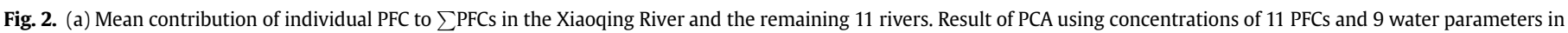
all rivers (b) and 11 rivers excluding the Xiaoqing River (c). 
scenarios of PCA (Fig. 2b and c), which indicated a probable coemission of these two PFSAs in the study area.

The profile of PFAAs showed that the south Bohai coastal rivers were mainly contaminated by PFOA followed by shorter chain PFCAs and PFOS. In recent studies, PFOA has been found to be the predominant PFAA in North Bohai coastal rivers (Wang et al., 2011b), rivers in Tianjin (Pan et al., 2011), Dianchi Lake (Zhang et al., 2012), Hanjiang River (Wang et al., 2013a), the Huaihe River Basin and Taihu Lake in China (Yu et al., 2012); Water samples from Hanoi city and its surrounding areas in Vietnam (Kim et al., 2013); Yodo River basin in Japan (Lien et al., 2008); the watershed of River Po in Northern Italy (Loos et al., 2008); and Mediterranean coastal rivers in Spain (Sánchez-Avila et al., 2010). However, only water in the Yodo and Po Rivers contained concentrations of PFOA that exceeded $1000 \mathrm{ng} / \mathrm{L}$, which was comparable to this study (Table S7). As far as we know, this is the highest concentration of PFOA in river water of China that has ever been reported.

\subsection{Spatial analysis of the fluoropolymer industry as the source}

High levels of PFOA implied local point sources in this area. As a result, the fluoropolymer industry in Shandong Province was investigated and the major manufacturing facilities were found located along the Xiaoqing River (Fig. 3), with production begun in 2001. The manufacturing history of the profile of PFAAs and related products in these facilities is unknown, but until now, fluorinated refrigerants, intermediates for production of pesticides and medicine, polytetrafluoroethylene (PTFE) and tetrafluoroethylene (TFE) have been the main products of these facilities. Facility 1 is the largest with an annual capacity of 37,000 tons of PTFE, 50,000 tons of TFE, 10,000 tons of hexafluoropropylene (HFP), and more than 200,000 tons of different types of fluorinated refrigerants by the end of 2012 (Fig. 4) (Dongyue Group Limited, 2012). The fluoropolymers production capacities of the other facilities ranged from hundreds to thousands of tons. PFOA is mainly produced and used as ammonium perfluorooctanoate (APFO) and further used as important processing additives for production of fluoropolymers and fluoroelastomers. For example, high purity APFO is used primarily in the dispersion polymerization process to produce PTFE. PTFE has unique properties like repellence to acid and alkali, thermal resistance, almost insoluble in solvents etc. Thus it has been used in many industrial and consumer products, including soil, stain, grease, and water resistant coatings on textiles and carpet; uses in the automotive, mechanical, aerospace, chemical, electrical, medical, and building/construction industries; personal care products; and non-stick coatings on cookware (European Commission, 2010). The emission of PFOA came from both the PTFE production discharge and applications of PTFE products (Fig. 4). Further study is needed to estimate the weights of the two ways for PFOA emissions. The fluorinated refrigerants (like R22), TFE and HFP are all important intermediates to produce PTFE and other fluoropolymers in different processes, with limited information on their emission of related PFAAs. While fluorinated ethylene propylene (FEP) is a modified material to PTFE and the production of FEP is still at the primary stage. Studies on these intermediates and new materials are also important not only to quantify known perfluoroalkyl and polyfluoroalkyl substances (PFASs), but also to qualify unknown species.

Spatial analysis of PFAAs levels, rivers, and production facilities together with the results obtained by other researchers indicated that facilities along the Xiaoqing River and its tributaries exhibited the greatest emissions of wastes, including sewage discharged

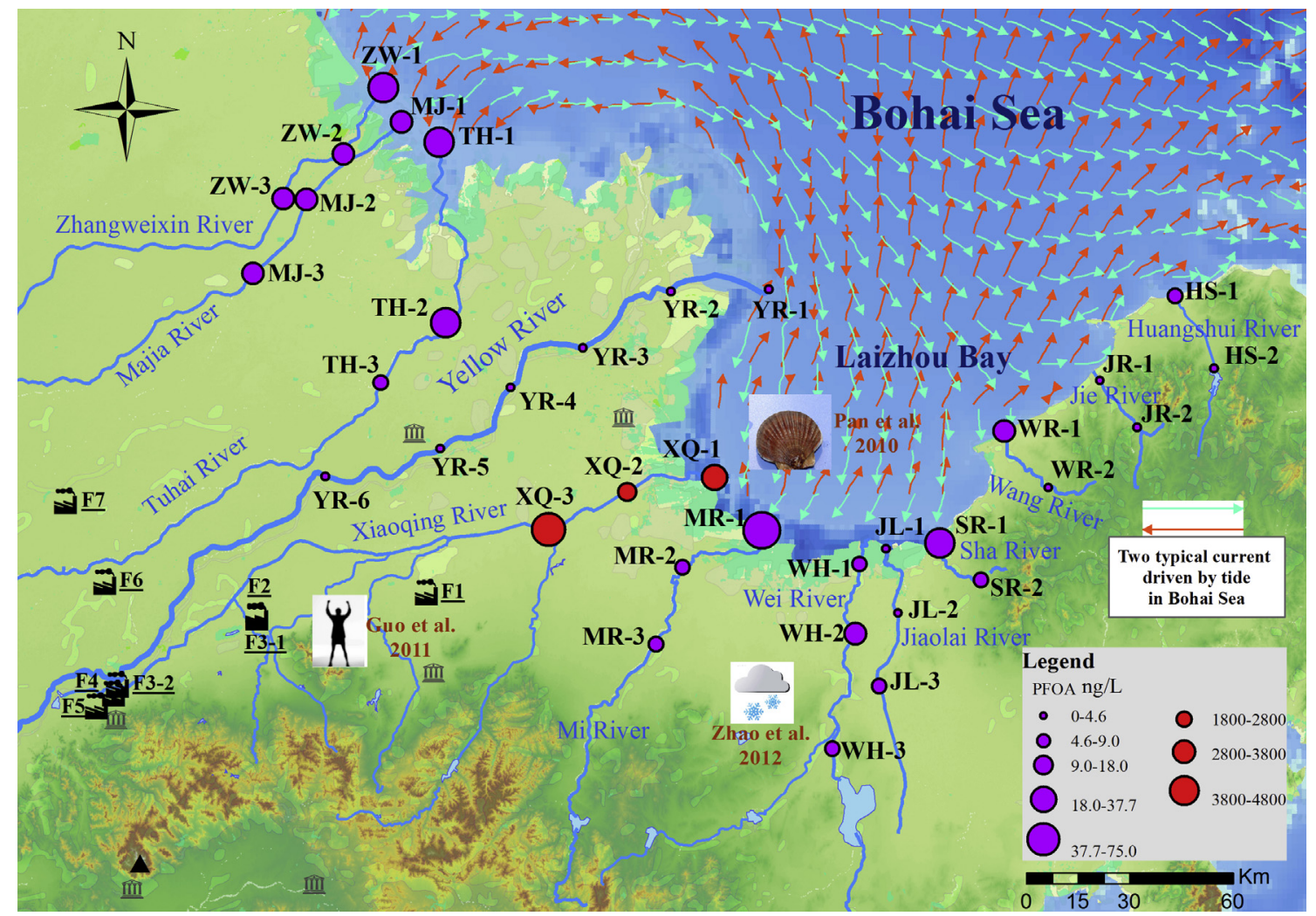

Fig. 3. Spatial distribution of PFOA in South Bohai coastal rivers, combined with available studies by other researchers and locations of manufacturing facilities. 


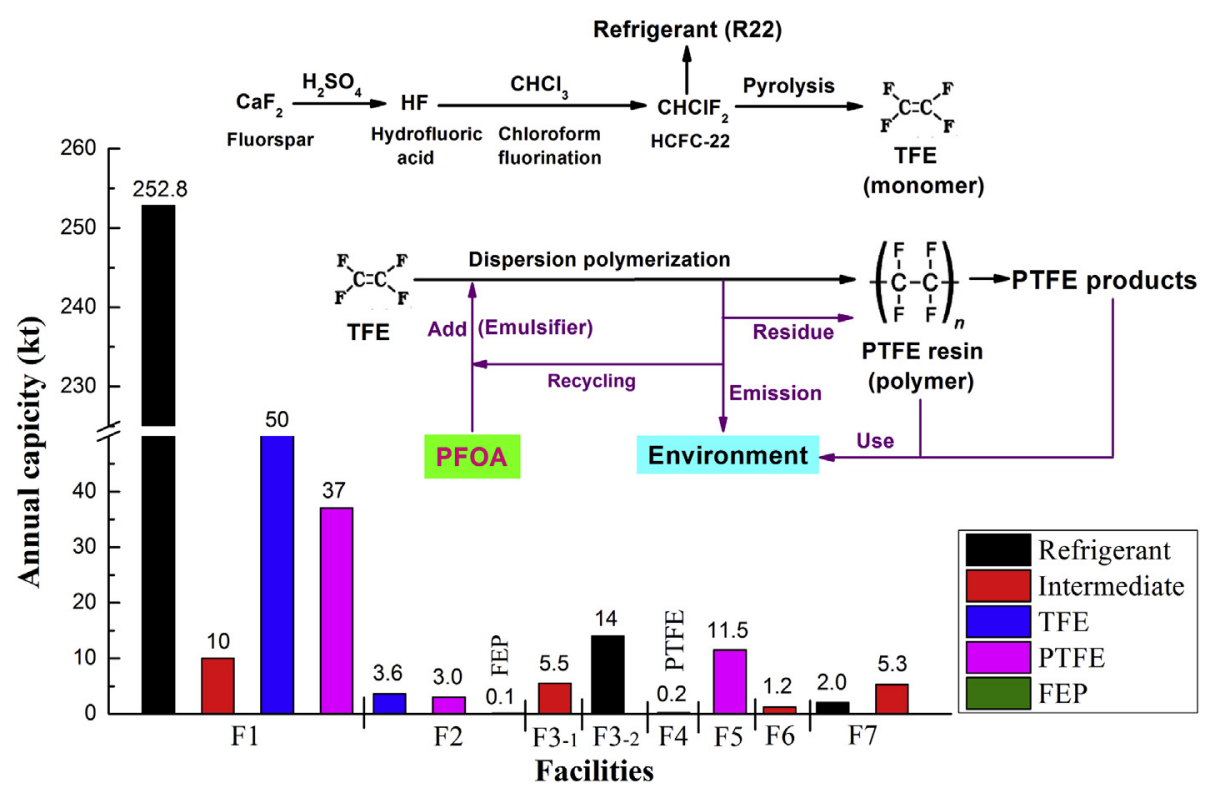

Fig. 4. Annual production capacity (kiloton, kt) of facilities surveyed in this study and the main industrial process for the production of TFE and PTFE.

directly to the river, and consequently resulted in the highest concentrations of PFOA measured in this study (Fig. 3). In addition, atmospheric transport and subsequent degradation of PFOA precursors including fluorotelomer alcohols (FTOHs) and perfluorooctane sulfonyl fluoride (POSF)-based chemicals (e.g., perfluorooctyl sulfonamidoethanols) could account for as much as $10 \%$ of PFOA emissions (Pistocchi and Loos, 2009). In a study conducted at one of 3M's largest fluoropolymer facilities in Minnesota (USA), WWTPs from both industrial and domestic discharges played a key role in the PFAAs releases to surface waters, and stormwater runoff from PFAAs-related commercial and industrial releases might also be a significant source of PFAAs to the surface water (Oliaei et al., 2013). So the significant correlations among PFCAs with carbon chain lengths from 4 to 8 in all rivers could be explained by the direct emission of waste from manufacturing facilities, atmospheric transport and degradation of precursors, input from WWTPs, or stormwater runoff, where the weights of these pathways might vary for different rivers. For the Yellow River, there are two main reasons for less concentrations of PFAAs, especially that of PFOA. One is that the riverbed of the section in Shandong Province has an average height of 4-6 $\mathrm{m}$ above the land surface, there is almost no possibility for it to receive waste from local facilities, and contribution from the upstream was also limited. The other is that the relatively greater water discharge compared to other rivers in this study would lead to more dilution (Table S1). Although in 2006, the eight major fluoropolymer and fluotelomer manufactures joined the US EPA 2010/2015 PFOA Stewardship Program working toward elimination of PFOA, its precursors and related higher homologue chemicals from emissions and their products by 2015 , facilities in this region as well as other facilities in China are still scaling up production to meet domestic and international demand without sufficient regulations on PFAAs emission (Wang et al., 2013b). The trend that PFOA levels were the highest among all PFAAs detected in this study was consistent with the results of other studies conducted in this area. Concentrations of PFOA in Mollusks were predominant with the highest concentration of $126 \mathrm{ng} / \mathrm{g}$ dry weight observed in Laizhou Bay, the estuary of Xiaoqing River, which was almost 40 times higher than that of PFOS (Pan et al., 2010). In the region of Zouping County where facilities 1 , 2 and 3-1 were located, the median concentration of PFOA in the whole human blood was $3.26 \mathrm{ng} / \mathrm{mL}$, while the median concentration of PFOS was $2.19 \mathrm{ng} / \mathrm{mL}$, which were the highest and the lowest among all cities investigated in Bohai Rim, respectively (Guo et al., 2011). PFOA was the dominant PFAA in precipitation across eastern and central China with a maximum concentration of $88 \mathrm{ng} /$ L observed in the city of Weifang (Zhao et al., 2013), where there is only small scale fluorinated chemical manufacturing compared to those in the Xiaoqing River basin. However, Tai'an city was also on the list of investigation in the precipitation study and had a much lower concentration of PFOA, but it is closer to the fluoropolymer manufacturing facilities than Weifang City. In this study, geomorphic analysis indicated that Mount Tai (the peak in Shandong Province) might be a natural block for the transport of volatile PFAAs in the atmosphere (Fig. S1). This result demonstrated that the mass loading of PFAAs for atmospheric deposition might be determined more by regional conditions than by local conditions. The major emission of PFOS in Shandong Province came from textile treatment and metal plating (Xie et al., 2013b). Unlike PFOA, release of PFOS from these kinds of manufactures is distributed more like non-point sources (Pistocchi and Loos, 2009), and no direct-emissions from these industries were observed in this study. Furthermore, PFOS and PFOA chemicals are still used in some pesticides with exceptions to the phase-out action. For example, sulfluramid will be phased-out by the year 2016 (Fluoride Action Network Pesticide project). In Shouguang County, which is the most famous production base of vegetables in China, although pesticide-free vegetables are dominating nowadays, the residues of PFAAs and their precursors that will degrade to PFAAs could be an issue for public health (Houde et al., 2011).

\subsection{Influence of waves and currents on dissipation of PFOA}

PFAAs undergo a mixing process and are dissipated by waves and currents when they move from the rivers to the sea. The process of dissipation in saline waters of estuaries when calculating the mass flux of PFAAs has been discussed previously (McLachlan et al., 2007). In this study, on a smaller scale, the effect of the estuarine drainage areas (EDA) showed more interesting characteristics of pollution dispersion within proximate rivers. Salinity was used to explain the extent to which water mixes (Fig. S3). However, it must 
be illustrated that the water was sampled in different times of the day while the tidal time varied daily, so the salinity only represented the status at the moment of sampling. The results showed that in all the rivers salinity in the EDA were greater than those at upstream locations despite the difference at the time of sampling. As an almost enclosed sea, the Bohai Sea has an estimated mixing time of approximately 30 years. For some of the small bays within the Bohai Sea, the turnover time could be even longer. It has been reported that poor exchange of seawater has led to accumulation of containments (i.e. chemical oxygen demand, heavy metals, nutrient salts) in these bays (Wang and Li, 2006), which indicated that in addition to the dilution process, part of the concentration of PFAAs in the EDA water might be contributed by intrusions of salty water, especially from adjacent rivers. The Yellow River represents a special case, in which the large discharge would make the estuary less influenced by seawater. When a numerical model was used to simulate wave-induced, near-shore currents and transport of pollutants in Bohai Bay, it was concluded that due to the action of waves in the near-shore zone with shallow water, pollutants were transported parallel to the shoreline (Sun and Tao, 2006). For the three rivers discharging into Bohai Bay, little influence on concentrations of PFOA was observed between the upstream and the EDA sites, so the influence of waves on these three rivers was not obvious. However in Laizhou Bay, in addition to greater salinity, concentrations of PFOA in EDA of the Mi, Sha, Wang and Huangshui Rivers were significantly higher than those at the upstream locations. In the Yellow, Xiaoqing, Wei, Jiaolai and Jia Rivers there was no significant difference in concentrations of PFOA as a function of salinity. This indicated that the majority of the PFOA at MR-1, SR-1, WR- 1 and HS-1 might come from XQ- 1 with the waves and currents along the shoreline. Although concentrations of PFOA were less at other EDA sites, they might increase notably with the rising tide. The ebb-tide (recession of sea level) in Laizhou Bay would lead to a current from the top of the bay eastward to HS-1 (Zhang, 2007), which is in good agreement with the results of the present study (Fig. 3). More attention should be paid to the influence of salinity changes arisen by waves and currents in the EDA as it could influence the property of PFAAs and also the physiology of organisms, which would consequently contribute to sorption and bioaccumulation of PFAAs in these organisms (Houde et al., 2011).

\subsection{Riverine input of PFAAs to the Bohai Sea}

Mass flux will provide information on the environmental inventory of PFAAs. In order to further eliminate the influence of residual seawater, sites used to calculate the mass flux of PFAAs were chosen based on salinity. Although the salinity of some EDA water was less than the freshwater threshold during ebb-tide, considering the frequent mixing, upstream sites of EDA were used to estimate loadings of PFAAs from rivers to the Bohai Sea. The mass flux was calculated based on instantaneous concentrations of PFAAs multiplied by the average annual water discharge data to give a rough yet valuable approximation (Table 1) (McLachlan et al., 2007; Filipovic et al., 2013). The mass flux of PFOA and $\sum$ PFAAs in the Xiaoqing River were 3.6 tons and 4 tons per year, which accounted for $90 \%$ and $80 \%$ of those among all the rivers, respectively. Even though the concentrations were less, there was the largest discharge in this study, and thus the Yellow River accounted for the second largest mass of $\sum$ PFAAs and PFOS, which were about 0.4 ton and 0.07 ton per year, respectively.

Excluding the extensive production capacity along the Xiaoqing River and the huge discharge of the Yellow River, mass flux of PFOS and PFOA into the Bohai Sea from the southern coastal rivers in this study was larger but still comparable to that of northern coastal rivers, which were calculated to be 0.02 and 0.2 ton per year for PFOS and PFOA, respectively. (Wang et al., 2011a) In comparison, the mass flux of PFOA from main rivers in the European Continent was estimated to be 14.3 tons per year (McLachlan et al., 2007). However, when averaged by area and population, the emission of PFOA in the South Bohai area was about 20 times greater than that in the European Continent, respectively, which posed a much heavier burden to the local environment.

\subsection{Assessment of risks}

Concentrations of PFOA in the Xiaoqing River exceeded several drinking water criteria including the New Jersey guidance for PFOA in drinking water $(40 \mathrm{ng} / \mathrm{L})$, the US EPA provisional health advisories for PFOA (400 ng/L), and the Health Canada drinking water guidance value for PFOA (700 ng/L) (New Jersey Department of Environmental Protection (NJDEP), 2007; USEPA, 2009; Paterson et al., 2012). The residents in the study area have not used the river water as drinking water for a long time. However, according to a study conducted on transport of PFOA near a fluoropolymer manufacturing facility, the atmospheric deposition would not only influence concentrations in surface waters, but also the underlying aquifer by migration downward with precipitation and river recharge (Davis et al., 2007). So there is still a large potential risk to the local drinking water system. Meanwhile, river water is used for irrigation, which might pose risks due to PFAAs in soils and subsequent accumulation into crops and vegetables and eventually accumulation in humans. None of the concentrations of PFOS or PFOA measured in this study exceeded any water quality criteria for the protection of freshwater aquatic organisms including criteria maximum concentration (CMC) and criteria continuous concentration (CCC). For example, the CMC was calculated to be $3.78 \mathrm{mg} / \mathrm{L}$

Table 1

Estimated mass flux of PFAAs in rivers to the Bohai Sea (kg/year).

\begin{tabular}{|c|c|c|c|c|c|c|c|c|c|c|c|c|}
\hline Rivers & PFBA & PFPeA & PFHxA & PFHpA & PFOA & PFNA & PFDA & PFUdA & PFBS & PFHxS & PFOS & $\sum$ PFAAs \\
\hline Zhangweixin River & 1.2 & 0.5 & 0.4 & 0.3 & 3.4 & 0.4 & 0.1 & $<0.1$ & $<0.1$ & $<0.1$ & 0.9 & 7.2 \\
\hline Majia River & 6.2 & 3.2 & 3.7 & 3.6 & 18.8 & 1.4 & 0.5 & 0.04 & 1.0 & 0.2 & 4.7 & 43.2 \\
\hline Tuhai River & 26.4 & 15.0 & 26.1 & 13.6 & 128.0 & 14.9 & 5.4 & 1.0 & 9.4 & $<0.1$ & 18.5 & 258.3 \\
\hline Yellow River & 120.2 & 17.8 & 21.7 & 14.7 & 131.9 & 20.3 & 1.3 & 0.6 & 25.6 & 10.4 & 69.3 & 433.9 \\
\hline Xiaoqing River & 63.8 & 84.7 & 147.6 & 115.6 & 3648.6 & 4.1 & 2.2 & 0.2 & 1.3 & 0.1 & 1.4 & 4069.6 \\
\hline Mi River & 1.2 & 0.4 & 0.6 & 0.5 & 3.9 & 0.3 & 0.1 & $<0.1$ & 0.1 & $<0.1$ & 1.4 & 8.6 \\
\hline Wei River & 24.2 & 4.5 & 6.2 & 5.8 & 37.0 & 6.8 & 2.3 & 0.3 & 0.7 & 2.2 & 38.0 & 127.8 \\
\hline Jiaolai River & 2.0 & 1.4 & 1.1 & 0.4 & 2.1 & 0.3 & 0.1 & $<0.1$ & $<0.1$ & 0.1 & 1.5 & 9.1 \\
\hline Sha River & 0.6 & 0.1 & 0.2 & 0.2 & 1.7 & 0.2 & $<0.1$ & $<0.1$ & $<0.1$ & $<0.1$ & 0.2 & 3.3 \\
\hline Wang River & $<0.1$ & $<0.1$ & $<0.1$ & $<0.1$ & $<0.1$ & $<0.1$ & $<0.1$ & $<0.1$ & $<0.1$ & $<0.1$ & 0.01 & 0.1 \\
\hline Jie River & 0.1 & $<0.1$ & $<0.1$ & $<0.1$ & 0.1 & $<0.1$ & $<0.1$ & $<0.1$ & $<0.1$ & $<0.1$ & 0.1 & 0.3 \\
\hline Huangshui River & 0.1 & $<0.1$ & $<0.1$ & $<0.1$ & 0.3 & $<0.1$ & $<0.1$ & $<0.1$ & $<0.1$ & $<0.1$ & $<0.1$ & 0.6 \\
\hline Total & 246.0 & 127.5 & 207.6 & 154.7 & 3975.9 & 48.7 & 12.1 & 2.2 & 38.2 & 13.1 & 136.0 & 4961.9 \\
\hline
\end{tabular}


for PFOS and $45.54 \mathrm{mg} / \mathrm{L}$ for PFOA, while CCC was $0.25 \mathrm{mg} / \mathrm{L}$ for PFOS and $3.52 \mathrm{mg} / \mathrm{L}$ for PFOA that were derived for the protection of freshwater aquatic life in China (Yang et al., 2014). The CMC for PFOS $(21 \mu \mathrm{g} / \mathrm{L})$ and PFOA (25 mg/L), CCC for PFOS $(5.1 \mu \mathrm{g} / \mathrm{L})$ and PFOA ( $2.9 \mathrm{mg} / \mathrm{L})$ and avian wildlife value (AWV) for PFOS (47 ng/L) were also derived based on toxicology data of organisms resident in North America (Giesy et al., 2010). When biodiversity in the main EDAs in China was investigated by use of the Shannon-Wiener index, Laizhou Bay exhibited relatively greater indices, indicative of good biodiversity and a constant environment (Huang et al., 2012), and was also consistent with the in-situ findings of this study. However, considering the almost lacking degradation property of PFOA, the scaling-up of its production, less water-mobility in Laizhou Bay and intensive fishery in this area, especially the aquaculture and salt fields in and around the coastal mudflat created by the rising tide (Fig. 3), further research is needed to evaluate the health risk of fish consumption for local residents to make sure that the pollution is controllable. Furthermore, the wave-induced near-shore current is usually considered to be the reason for sediment suspension in the near-shore zone (Sun and Tao, 2006), and many aquatic organisms ingest particles in water (Jeon et al., 2010). This would increase the bioconcentration factors of the aquatic ecosystem and also the overall risk.

\section{Conclusions}

The present study gave a general characterization of the PFAAs pollution in the main rivers of the rapidly urbanized South Bohai coastal region with PFOA dominant in pretty high concentration. Previous studies on source identification would use a combination of geographic information, such as population density and land use as indicators for the influence of urbanization on PFAAs emissions, especially for PFOS (Murakami et al., 2008; Pistocchi and Loos, 2009; Zushi and Masunaga, 2009). Because the majority of the PFOA loads are emitted from industries, distributions of fluoropolymer industry in Shandong Province were investigated and the main facilities were identified so that monitoring could be conducted to establish the distribution, status and trend in concentrations of PFAAs in this region. The data on the concentrations of PFAAs was also combined with GIS data including land and sea DEM, and vegetation data to provide a visual description of contamination in the region. For China, the scaling-up of fluoropolymer production would predictably bring actions on risk assessment and regulation in the future, and results in this study would provide valuable information. It may also provide hint for other countries with rapid urbanization to take precautionary approach to tackling the emerging pollution.

\section{Acknowledgments}

This study was supported by the National Natural Science Foundation of China under Grant No. 41371488 and 41171394, the International Scientific Cooperation Program with Grant No. 2012DFA91150, and the Key Project of the Chinese Academy of Sciences under Grant No. KZZD-EW-TZ-12. We would like to thank the editors and reviewers for their valuable comments and suggestions. Prof. Giesy was supported by the Einstein Professor Program of the Chinese Academy of Sciences and the Canada Research Chair program.

\section{Appendix A. Supplementary data}

Supplementary data related to this article can be found at http:// dx.doi.org/10.1016/j.envpol.2014.03.030.

\section{References}

Armitage, J.M., MacLeod, M., Cousins, I.T., 2009. Comparative assessment of the global fate and transport pathways of long-chain perfluorocarboxylic acids (PFCAs) and perfluorocarboxylates (PFCs) emitted from direct sources. Environ. Sci. Technol. 43 (15), 5830-5836.

Bao, J., Jin, Y.H., Liu, W., Ran, X.R., Zhang, Z.X., 2009. Perfluorinated compounds in sediments from the Daliao River system of northeast China. Chemosphere 77 (5), 652-657.

Bao, J., Liu, W., Liu, L., Jin, Y.H., Dai, J.Y., Ran, X.R., Zhang, Z.X., Tsuda, S., 2010. Perfluorinated compounds in the environment and the blood of residents living near fluorochemical plants in Fuxin, China. Environ. Sci. Technol. 45 (19), 8075-8080.

Chen, C.L., Lu, Y.L., Zhang, X., Geng, J., Wang, T.Y., Shi, Y.J., Hu, W.Y., Li, J., 2009. A review of spatial and temporal assessment of PFOS and PFOA contamination in China. Chem. Ecol. 25 (3), 163-177.

Chen, C.L., Wang, T.Y., Naile, J.E., Li, J., Geng, J., Bi, C.C., Hu, W.Y., Zhang, X., Khim, J.S., Feng, Y., Giesy, J.P., Lu, Y.L., 2011. Perfluorinated compounds in aquatic products from Bohai Bay, Tianjin, China. Hum. Ecol. Risk Assess. Int. J. 17 (6), 1279-1291.

Davis, K.L., Aucoin, M.D., Larsen, B.S., Kaiser, M.A., Hartten, A.S., 2007. Transport of ammonium perfluorooctanoate in environmental media near a fluoropolymer manufacturing facility. Chemosphere 67 (10), 2011-2019.

Dongyue Group Limited, 2012. 2012 Annual Report. http://www.dongyuechem. com/UploadFiles/2013-3/News/20133201940820043.pdf (March 10 2014).

European Commission, 2010. Analysis of the Risks Arising from the Industrial Use of Perfuorooctanoic Acid (PFOA) and Ammonium Perfluorooctanoate (APFO) and from Their Use in Consumer Articles. Evaluation of the Risk Reduction Measures for Potential Restrictions on the Manufacture, Placing on the Market and Use of PFOA and APFO. http://ec.europa.eu/enterprise/sectors/chemicals/files/docs_ studies/final_report_pfoa_pfos_en.pdf (March 10 2014).

Filipovic, M., Berger, U., McLachlan, M.S., 2013. Mass balance of perfluoroalkyl acids in the Baltic Sea. Environ. Sci. Technol. 47 (9), 4088-4095.

Fluoride Action Network Pesticide Project. http://www.fluoridealert.org/pesticides/ pfos.pfoas-page.htm (March 10 2014).

Giesy, J.P., Kannan, K., 2002. Peer reviewed: perfluorochemical surfactants in the environment. Environ. Sci. Technol. 36 (7), 146A-152A.

Giesy, J.P., Mabury, S.A., Martin, J.W., Kannan, K., Jones, P.D., Newsted, J.L., Coady, K., 2006. Perfluorinated compounds in the Great Lakes. Persistent Org. Pollut. Gt. Lakes 5, 391-438.

Giesy, J.P., Naile, J.E., Khim, J.S., Jones, P.D., Newsted, J.L., 2010. Aquatic toxicology of perfluorinated chemicals. In: Whitacre, D.M. (Ed.), Reviews of Environmental Contamination and Toxicology, vol. 202. Springer, New York, pp. 1-52.

Guo, F.F., Zhong, Y.X., Wang, Y.X., Li, J.G., Zhang, J.L., Liu, J.Y., Zhao, Y.F., Wu, Y.N., 2011 Perfluorinated compounds in human blood around Bohai Sea, China. Chemosphere 85 (2), 156-162.

Houde, M., De Silva, A.O., Muir, D.C.G., Letcher, R.J., 2011. Monitoring of perfluorinated compounds in aquatic biota: an updated review. Environ. Sci. Technol. 45 (19), 7962-7973.

Huang, J.L., Li, Q.S., Huang, L., Wang, J.Y., Hu, Y.Y., Feng, Y., 2012. Preliminary delineation and classification of estuarine drainage areas for major coastal rivers in China. Acta Ecol. Sin. 32 (11), 3516-3527.

Jeon, J., Kannan, K., Lim, H.K., Moon, H.B., Ra, J.S., Kim, S.D., 2010. Bioaccumulation of perfluorochemicals in Pacific Oyster under different salinity gradients. Environ. Sci. Technol. 44 (7), 2695-2701.

Jin, Y.H., Saito, N., Harada, K.H., Inoue, K., Koizumi, A., 2007. Historical trends in human serum levels of perfluorooctanoate and perfluorooctane sulfonate in Shenyang, China. Tohoku J. Exp. Med. 212 (1), 63-70.

Kim, J.-W., Tue, N., Isobe, T., Misaki, K., Takahashi, S., Viet, P., Tanabe, S., 2013. Contamination by perfluorinated compounds in water near waste recycling and disposal sites in Vietnam. Environ. Monit. Assess. 185 (4), 2909-2919.

Li, F.S., Sun, H.W., Hao, Z.N., He, N., Zhao, L.J., Zhang, T. Sun, T.H., 2011. Perfluorinated compounds in Haihe River and Dagu drainage Canal in Tianjin, China. Chemosphere 84 (2), 265-271.

Lien, N.P.H., Fujii, S., Tanaka, S., Nozoe, M., Tanaka, H., 2008. Contamination of perfluorooctane sulfonate (PFOS) and perfluorooctanoate (PFOA) in surface water of the Yodo River basin (Japan). Desalination 226 (1-3), 338-347.

Liu, W., Jin, Y.H., Quan, X., Sasaki, K., Saito, N., Nakayama, S.F., Sato, I., Tsuda, S., 2009. Perfluorosulfonates and perfluorocarboxylates in snow and rain in Dalian, China. Environ. Int. 35 (4), 737-742.

Loos, R., Locoro, G., Huber, T., Wollgast, J., Christoph, E.H., de Jager, A., Manfred Gawlik, B., Hanke, G., Umlauf, G., Zaldivar, J.M., 2008. Analysis of perfluorooctanoate (PFOA) and other perfluorinated compounds (PFCs) in the River Po watershed in N-Italy. Chemosphere 71 (2), 306-313.

McLachlan, M.S., Holmström, K.E., Reth, M., Berger, U., 2007. Riverine discharge of perfluorinated carboxylates from the European Continent. Environ. Sci. Technol. 41 (21), 7260-7265.

Moller, A., Ahrens, L., Surm, R., Westerveld, J., van der Wielen, F., Ebinghaus, R., de Voogt, P., 2010. Distribution and sources of polyfluoroalkyl substances (PFAS) in the River Rhine watershed. Environ. Pollut. 158 (10), 3243-3250.

Muller, C.E., Spiess, N., Gerecke, A.C., Scheringer, M., Hungerbuhler, K., 2011. Quantifying diffuse and point inputs of perfluoroalkyl acids in a nonindustrial river catchment. Environ. Sci. Technol. 45 (23), 9901-9909.

Murakami, M., Imamura, E., Shinohara, H., Kiri, K., Muramatsu, Y., Harada, A., Takada, H., 2008. Occurrence and sources of perfluorinated surfactants in rivers in Japan. Environ. Sci. Technol. 42 (17), 6566-6572. 
New Jersey Department of Environmental Protection (NJDEP), 2007. Guidance for PFOA in Drinking Water at Pennsgrove Water Supply Company. http://www.nj. gov/dep/watersupply/pdf/pfoa_dwguidance.pdf (March 10 2014).

Oliaei, F., Kriens, D., Weber, R., Watson, A., 2013. PFOS and PFC releases and associated pollution from a PFC production plant in Minnesota (USA). Environ. Sci. Pollut. Res. 20 (4), 1977-1992.

Pan, Y.Y., Shi, Y.L., Wang, J.M., Jin, X.L., Cai, Y.Q., 2011. Pilot investigation of perfluorinated compounds in river water, sediment, soil and fish in Tianjin, China. Bull. Environ. Contam. Toxicol. 87 (2), 152-157.

Pan, Y.Y., Shi, Y.L., Wang, Y.W., Cai, Y.Q., Jiang, G.B., 2010. Investigation of perfluorinated compounds (PFCs) in mollusks from coastal waters in the Bohai Sea of China. J. Environ. Monit. 12 (2), 508-513.

Paterson, L., Mitchell, I., Chatwell, I., Birk, R., 2012. Evaluation of Groundwater Transport of Perfluorinated Chemicals at a Former Fire-Fighting Training Area. http://www.rpic-ibic.ca/documents/2012_fcs_presentations/Paterson_E.pdf (March 10 2014).

Pistocchi, A., Loos, R., 2009. A map of European emissions and concentrations of PFOS and PFOA. Environ. Sci. Technol. 43 (24), 9237-9244.

Prevedouros, K., Cousins, I.T., Buck, R.C., Korzeniowski, S.H., 2005. Sources, fate and transport of perfluorocarboxylates. Environ. Sci. Technol. 40 (1), 32-44.

Sánchez-Avila, J., Meyer, J., Lacorte, S., 2010. Spatial distribution and sources of perfluorochemicals in the NW mediterranean coastal waters (Catalonia, Spain). Environ. Pollut. 158 (9), 2833-2840.

Sun, T., Tao, J.H., 2006. Numerical simulation of pollutant transport acted by wave for a shallow water sea bay. Int. J. Numer. Methods Fluids 51 (5), 469-487.

Taniyasu, S., Kannan, K., So, M.K., Gulkowska, A., Sinclair, E., Okazawa, T., Yamashita, N., 2005. Analysis of fluorotelomer alcohols, fluorotelorner acids, and short- and long-chain perfluorinated acids in water and biota. J. Chromatogr. A 1093 (1-2), 89-97.

USEPA, 2009. Provisional Health Advisories for Perfluorooctanoic Acid (PFOA) and Perfluorooctane Sulfonate (PFOS). http://water.epa.gov/action/advisories/ drinking/upload/2009_01_15_criteria_drinking_pha-PFOA_PFOS.pdf (March 10 2014).

Wang, B., Cao, M., Zhu, H., Chen, J., Wang, L., Liu, G., Gu, X., Lu, X., 2013a. Distribution of perfluorinated compounds in surface water from Hanjiang River in Wuhan, China. Chemosphere 93 (3), 468-473.

Wang, P., Wang, T.Y., Giesy, J.P., Lu, Y.L., 2013b. Perfluorinated compounds in soils from Liaodong Bay with concentrated fluorine industry parks in China. Chemosphere 91 (6), 751-757.
Wang, T.Y., Khim, J.S., Chen, C.L., Naile, J.E., Lu, Y.L., Kannan, K., Park, J., Luo, W., Jiao, W.T., Hu, W.Y., Giesy, J.P., 2011a. Perfluorinated compounds in surface waters from Northern China: comparison to level of industrialization. Environ. Int. 42, 37-46.

Wang, T.Y., Lu, Y.L., Chen, C.L., Naile, J.E., Khim, J.S., Park, J., Luo, W., Jiao, W.T, Hu, W.Y., Giesy, J.P., 2011b. Perfluorinated compounds in estuarine and coastal areas of north Bohai Sea, China. Mar. Pollut. Bull. 62 (8), 1905-1914.

Wang, X.L., Li, K.Q., 2006. Marine Environmental Capacity of Pollutants in Bohai Sea. Science Press, China.

Xie, S., Lu, Y., Wang, T., Liu, S., Jones, K., Sweetman, A., 2013a. Estimation of PFOS emission from domestic sources in the eastern coastal region of China. Environ. Int. 59 (0), 336-343.

Xie, S.W., Wang, T.Y., Liu, S.J., Jones, K.C., Sweetman, A.J., Lu, Y.L., 2013b. Industrial source identification and emission estimation of perfluorooctane sulfonate in China. Environ. Int. 52 (0), 1-8.

Yang, S., Xu, F., Wu, F., Wang, S., Zheng, B., 2014. Development of PFOS and PFOA criteria for the protection of freshwater aquatic life in China. Sci. Total Environ. 470-471 (0), 677-683.

Yu, N.Y., Shi, W., Zhang, B.B., Su, G.Y., Feng, J.F., Zhang, X.W., Wei, S., Yu, H.X., 2012 Occurrence of perfluoroalkyl acids including perfluorooctane sulfonate isomers in Huai River Basin and Taihu Lake in Jiangsu Province, China. Environ. Sci. Technol. 47 (2), 710-717.

Zhang, N., 2007. Bohai Sea Tide Forecasting and Characteristic Analysis of Flow-Field (in Chinese). Tianjin University.

Zhang, Y., Meng, W., Guo, C., Xu, J., Yu, T., Fan, W., Li, L., 2012. Determination and partitioning behavior of perfluoroalkyl carboxylic acids and perfluorooctanesulfonate in water and sediment from Dianchi Lake, China. Chemosphere 88 (11), 1292-1299.

Zhao, L.J., Zhou, M., Zhang, T., Sun, H.W., 2013. Polyfluorinated and perfluorinated chemicals in precipitation and runoff from cities across Eastern and Central China. Arch. Environ. Contam. Toxicol. 64 (2), 198-207.

Zushi, Y., Masunaga, S., 2009. Identifying the nonpoint source of perfluorinated compounds using a geographic information system based approach. Environ. Toxicol. Chem. 28 (4), 691-700.

Zushi, Y., Ye, F., Motegi, M., Nojiri, K., Hosono, S., Suzuki, T., Kosugi, Y., Yaguchi, K. Masunaga, S., 2012. Spatial distribution and loading amounts of particle sorbed and dissolved perfluorinated compounds in the basin of Tokyo Bay. Chemosphere 88 (11), 1353-1357. 